\title{
Cardiorenal Syndromes: Pathophysiology to Prevention
}

\author{
Peter A. McCullough \\ Department of Medicine, Cardiology Section, St. John Providence Health System, Providence Park Heart Institute, \\ 47601 Grand River Avenue, Suite C202, Novi, MI 48374, USA
}

Correspondence should be addressed to Peter A. McCullough, peteramccullough@gmail.com

Received 21 August 2010; Accepted 30 September 2010

Academic Editor: Mitchell H. Rosner

Copyright () 2011 Peter A. McCullough. This is an open access article distributed under the Creative Commons Attribution License, which permits unrestricted use, distribution, and reproduction in any medium, provided the original work is properly cited.

\begin{abstract}
There is a strong association between both acute and chronic dysfunction of the heart and kidneys with respect to morbidity and mortality. The complex interrelationships of longitudinal changes in both organ systems have been difficult to describe and fully understand due to a lack of categorization of the common clinical scenarios where these phenomena are encountered. Thus, cardiorenal syndromes (CRSs) have been subdivided into five syndromes which represent clinical vignettes in which both the heart and the kidney are involved in bidirectional injury and dysfunction via a final common pathway of cell-to-cell death and accelerated apoptosis mediated by oxidative stress. Types 1 and 2 involve acute and chronic cardiovascular disease (CVD) scenarios leading to acute kidney injury (AKI) or accelerated chronic kidney disease (CKD). Types 3 and 4 describe AKI and CKD, respectively, leading primarily to heart failure, although it is possible that acute coronary syndromes, stroke, and arrhythmias could be CVD outcomes in these forms of CRS. Finally, CRSs type 5 describe a systemic insult to both heart and the kidneys, such as sepsis, where both organs are injured simultaneously in persons with previously normal heart and kidney function at baseline. Both blood and urine biomarkers, including the assessment of catalytic iron, a critical element to the generation of oxygen-free radicals and oxidative stress, are reviewed in this paper.
\end{abstract}

\section{Introduction}

The worldwide pandemic of excess adiposity is the "common soil" for mutual risk factors leading to cardiovascular disease (CVD) and chronic kidney disease (CKD) including the metabolic syndrome, diabetes, hypertension, dyslipidemia, neurohormonal activation, and systemic inflammation. Both cardiac and renal diseases commonly present in the same patient and have been associated with increased cost of care, complications, and mortality $[1,2]$. There is an immediate and present need to categorize the complex relationships between acute and chronic organ injury and dysfunction that exist with respect to the heart and kidneys. The cardiorenal syndromes (CRSs) describe the dynamic interrelationship between heart and kidney malfunction and have been clarified in a recent consensus effort led by the Acute Dialysis Quality Initiative (ADQI) [3]. Five distinct CRSs have been proposed. This paper will review this new classification scheme and giving vignettes of each syndrome discuss available information on recognition and management. In addition, a targeted review of promising biomarkers will be presented. It is expected that these biomarkers will considerably enhance the current body of literature concerning CRSs which is largely based on single blood biomarker-serum creatinine and its derivative, the estimated glomerular filtration rate (eGFR).

\section{Five Cardiorenal Syndromes}

The plural term CRSs suggests several subtypes denoted by the principal organ dysfunction by temporal sequence (cardiac versus renal or simultaneous) as well as the relative acuity of each illness. Both organs must have or develop evidence of pathological changes to fulfill the criteria for definition. The umbrella term "cardiorenal syndromes" was defined as "Disorders of the heart and kidneys whereby acute or chronic dysfunction in one organ may induce acute or chronic dysfunction of the other" [3]. Figure 1 displays an array of possible pathophysiologic mechanisms for each 

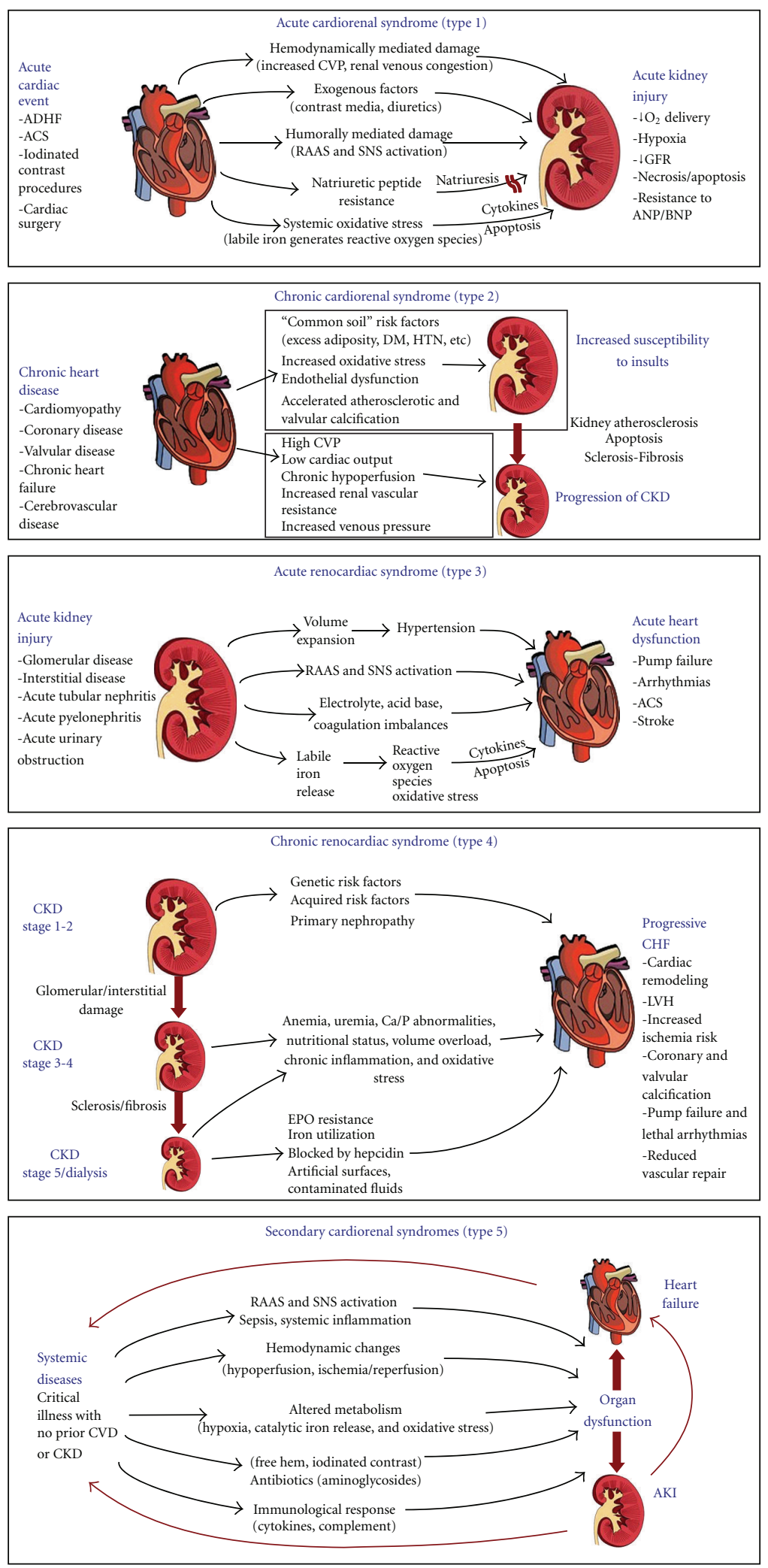

FIGURE 1: Pathophysiology and definitions of the five subtypes of cardiorenal syndromes. 
syndrome. As shown, it has been recently understood that the process of oxidative stress resulting in cell dysfunction, accelerated apoptosis, and death is reliant on the cytosolic and extracellular presence of labile or catalytic iron. There are several steps in generation of reactive oxygen species (ROS). Oxygen may be reduced forming superoxide anion, which can undergo reduction by superoxide dismutase to form hydrogen peroxide which itself can then be reduced through several pathways. The net reaction is slow and in the presence of reduced transition metals such as ferric iron $\left(\mathrm{Fe}^{+3}\right)$, a Haber-Weiss reaction results in the rapid formation of the highly damaging hydroxyl radical from the superoxide anion. Likewise, in the presence of ferrous iron $\left(\mathrm{Fe}^{+2}\right)$, a Fentontype reaction converts hydrogen peroxide to the hydroxyl radical. Thus, the presence of labile iron, in excess both in the cytosol and in the extracellular space, could result in the generation of the hydroxyl radical which further causes destruction of cellular organelles and membranes. Further reduction of hydroxyl radical finally ends in the formation of water. It has been theorized that a common element to all forms of oxidative stress to the heart and kidneys involves the periodic availability of unbound or poorly liganded iron [4]. There is a complex management system for iron metabolism keeping it bound in transport proteins, heme, and cellular organelles for normal functioning $[5,6]$. If small amounts of iron are released from adjacent injured cells and not immediately bound, this poorly liganded (labile or catalytic) iron in either the ferric or ferrous states facilitates the rapid generation of oxygen-free radicals and the propagation of oxidative stress and injury across regions of vascular tissue [7]. Therefore, a putative final common pathway for common sources of organ injury resulting in CRSs including ischemia, neurohormonal activation, chemotoxicity, and sepsis involves the loss of control over normal iron management and the transient tissue and organ system exposure to catalytic iron.

2.1. Acute Cardiorenal Syndrome (Type 1): Acute Cardiac Event Precipitating AKI. This is a syndrome of worsening renal function that frequently complicates acute decompensated heart failure (ADHF) and acute coronary syndrome (ACS). Seven observational studies have reported on the frequency and outcomes of CRSs Type 1 in the setting of ADHF and five in ACS [8]. Approximately one-third of patients hospitalized for ADHF develop acute kidney injury (AKI) as defined by an increase in serum creatinine of $\geq 0.3 \mathrm{mg} / \mathrm{dl}[8,9]$. Baseline CKD, diabetes, prior HF, and initial presentation with hypertension are established risk predictors for CRSs Type 1 [10]. Complicated hospital courses with hemodynamic decompensation, longer inpatient stays, and higher mortality have all been consistently described with CRSs Type 1. However, part of this relationship can be attributed to confounding by temporal association as observed by the Prospective Outcomes Study in Heart Failure (POSH) study, where only ADHF cases with a rise in serum creatinine $(\geq 0.3 \mathrm{mg} / \mathrm{dl})$ who concurrently developed hemodynamic compromise, cardiac arrest, infection, or acute coronary ischemia were observed to have a higher six-month mortality [11]. Conversely, those with a similar rise in serum creatinine but no other complications did not incur higher death rates in the hospital, at 30 or 180 days compared to those without such a rise in creatinine. Because CRSs Type 1 in patients with heart failure rarely occurs in the prehospital phase and more commonly develops after treatment is started in hospital, iatrogenic factors have been implicated. The use of loop diuretics, probably by further activating the renin-angiotensin system and possibly worsening intrarenal hemodynamics, has been identified as one of the modifiable in-hospital determinants of CRSs Type 1 [12]. In the Evaluation Study of Congestive Heart Failure and Pulmonary Artery Catheterization Effectiveness (ESCAPE) trial, the use of higher doses of loop diuretics, causing hemoconcentration, resulted in a 5-fold increased rate of worsening renal function [13]. However, it should be noted despite these observations that aggressive diuresis was associated with a $69 \%$ reduction in death at 180 days. The presence of an elevated central venous pressure and inferred renal venous congestion, as opposed to hypotension or poor cardiac output, has been associated with the development of CRSs Type 1. The relative balance of arterial and venous pressure, volume, and flow resulting in congestion of the kidney appear to be important in the drop in renal filtration that occurs during acute treatment of AHDF [14].

Another scenario where CRSs Type 1 has been described is in the setting of coronary revascularization procedures. Acute contrast-induced and cardiopulmonary bypass surgery-associated AKI has been reported in approximately 15 and $30 \%$ of patients, respectively $[15,16]$. Iodinated contrast causes transient renal vasoconstriction, and medullary hypoxia and is directly chemotoxic to renal tubular cells. Its use is the primary cause of AKI in the setting of ACS and elective coronary catheter-based procedures. In addition, contrast-induced acute kidney injury (CI-AKI) is an important pre-existing factor in the prior days before cardiac surgery rendering patients at risk for further renal injury with cardiopulmonary bypass. Cardiac surgery exposes the kidneys to hypothermic, pulseless reduced perfusion for 3090 minutes and results in reduced renal perfusion in the setting of a proinflammatory state [17]. The extracorporeal circuit used in cardiopulmonary bypass surgery activates systemic factors and may release catalytic iron from heme, which works to induce AKI in the setting of reduced temperature and flow to the kidneys [18]. Reducing exposure to the perfusion circuit has not resulted in reduced rates of AKI but has attenuated its severity [19]. Contrastinduced AKI and cardiac surgery-associated AKI are in a temporal pathophysiologic sequence since almost every cardiac surgery patient operated upon in the urgent setting undergoes coronary angiography in the hours to days before surgery [20]. Similar to ADHF, patients undergoing revascularization with complications, CRSs Type 1 appears to be independently associated with a 3-to 4 -fold increase in mortality irrespective of the use of renal replacement therapy $[21,22]$. In all forms of CRSs Type 1 , there is a risk of advancing to higher stages of CKD and end-stage renal disease (ESRD) [23]. The incremental and cumulative risk of these renal outcomes in patients undergoing multiple 
coronary revascularization procedures over a lifetime is not known. Salient features of CRSs Type 1 described in the literature to date include (1) higher mortality risk can be attributed to nonrenal complications (shock, infection, and arrhythmias) occurring during the hospitalization and not the rise in creatinine itself, (2) intravascular iodinated contrast alone, and in conjunction with cardiopulmonary bypass, initiates AKI via a transient reduction in renal blood flow and medullary hypoxia followed by direct chemotoxicity to renal tubular cells, and (3) during ADHF hospitalization, the use of iodinated contrast or other cardiac procedures is associated with longer lengths of stay and higher mortality which is possibly in part attributable to CRSs Type 1 [24-26].

Preventive Approaches. It is beyond the scope of this paper to review the body of clinical trials that have attempted to reduce contrast-induced and cardiac surgery-associated AKI. The basic principles include avoidance of volume depletion, removal of superimposed renal toxic agents (nonsteroidal anti-inflammatory agents, aminoglycosides), minimization of the toxic exposure (iodinated contrast, time on cardiopulmonary bypass), and possibly the use of antioxidant agents such as $\mathrm{N}$-acetylcysteine (for contrast exposure) and B-type natriuretic peptide in the perioperative period after cardiac surgery [27, 28]. More broadly across all forms of CRSs Type 1 , consideration should be given for forms of continuous renal replacement therapy (CRRT) in the period of time surrounding the renal insult. Conceptually, the use of CRRT provides three important protective mechanisms that cannot be achieved pharmacologically as follows: (1) it ensures euvolemia and avoids hypo- or hypervolemia, (2) it provides sodium and solute (nitrogenous waste products) removal, and (3) by both mechanisms above, it may work to avoid both passive renal congestion and a toxic environment for the kidneys and allow their optimal function during a systemically vulnerable period [29]. Despite these advantages, there remains a lack of clinical trial data supporting CRRT over other forms of extracorporeal solute removal. Finally, for patients in whom anuria and serious renal failure have a high probability of occurring, the upstream use of CRRT removes the hazards around the critical period of initiation of dialysis including electrolyte imbalance, urgent catheter placement, and extreme volume overload.

\subsection{Chronic Cardiorenal Syndrome (Type 2): Chronic CVD} Leading to Progressive CKD. It is important to recognize that chronic CVD in some cases leads to the progression of CKD. Observational studies have suggested that CVD contributes to an excess risk of CKD and its progression (CRSs Type 2) [8]. The established risk factors for atherosclerosis, namely diabetes, hypertension, and smoking are independently associated with the development of CKD [30]. Left ventricular systolic and diastolic dysfunction can lead to alterations in neurohormonal activation, renal hemodynamics, and a variety of adverse cellular processes leading to apoptosis and renal fibrosis [31]. One-third of the prevalent pool of CVD has concurrent CKD and, when combined, leads to further disease progression [29]. In the National Kidney
Foundation, Kidney Early Evaluation Program, CKD has been associated with premature CVD events including MI and stroke $[32,33]$. Chronic kidney disease-associated bone and mineral disorder characterized by phosphate retention, relative vitamin D deficiency and calcium availability, and secondary hyperparathyroidism is pathophysiology linked to the accelerated calcific atherosclerosis observed in patients with CKD [34]. Hyperphosphatemia, due to phosphate retention, stimulates the conversion of vascular smooth muscle cells to osteoblastic-like cells which, via the Pit-1 receptor, are stimulated to produce extracellular calcium hydroxyapatite crystals in the vascular smooth muscle layer of atherosclerotic arteries $[35,36]$. Thus, patients as a part of CRSs type 2 more commonly have vascular calcification, less vascular compliance, and a higher degree of chronic organ injury due to shear stress at the large, medium, and smaller vessel levels [37]. Despite these mechanisms specific to CRSs, CRSs Type 2 remains heavily confounded by the "common soil" of atherosclerosis and CKD. Excess adiposity and the cardiometabolic syndrome with activation of the sympathetic and renin-angiotensin systems as well as adipokine-stimulated systemic inflammation affect both organ systems; therefore, it is likely that for most patients with CRSs Type 2, concurrent organ injury is occurring based on these pathophysiologic mechanisms [38].

Approximately half of chronic HF patients have evidence of CKD defined as an estimated glomerular filtration rate (eGFR) $<60 \mathrm{ml} / \mathrm{min} / 1.73 \mathrm{~m}^{2}$ [39]. The presence of CKD has been associated with more frequent hospitalizations and death from pump failure and arrhythmias [40, 41]. Sodium and water retention are the primary cardiac insults put forward as to how CKD worsens HF. It should also be mentioned that CKD is associated with diuretic resistance and higher rates of drug intolerance to inhibitors of the renin-angiotensin-aldosterone system. Patients with CKD and ESRD have greater risks of ventricular arrhythmias and higher defibrillation thresholds; thus, they may have higher failure rates of antiarrhythmic therapy and implantable cardiodefibrillators [42]. Greater left ventricular mass and cardiac fibrosis may be responsible electrophysiological findings in patients with CKD [43]. Finally, asymptomatic cerebral infarctions by magnetic resonance imaging have been associated with a rapid decline in renal function in approximately $30 \%$ of patients [44]. This suggests the possibility that systemic atherosclerosis contributes to more rapid progression of CKD. Future research on the systemic and direct effects of atherosclerosis on the kidney is needed.

Preventive Approaches. As a general axiom, pharmacologic therapies that have been beneficial for chronic CVD have been either neutral or favorable to the kidneys including use of renin angiotensin aldosterone system (RAAS) antagonists, beta-adrenergic blocking agents, and statins. Furthermore, other strategies which are modestly beneficial from a cardiac perspective have even a larger benefit on microvascular injury to the kidneys including glycemic control in diabetes and blood pressure control in those with hypertension [45]. Finally, there is some support from clinical trials that fibric acid derivatives may preferentially reduce rates of 
microalbuminuria in patients with CKD. The long-term clinical implications of these observations are unknown [46].

2.3. Acute Cardiorenal Syndrome (Type 3): Acute Worsening of Renal Function Leading to Cardiac Events. A well-described vignette for CRSs Type 3 is the development of AKI resulting in volume overload, sodium retention, neurohormonal activation, and ADHF with the cardinal features fatigue, breathlessness, and peripheral edema. In children, isolated volume overload has been shown to induce myocardial dysfunction and CRSs Type 3 [47]. The picture is not so clear in adults, when acute on chronic disease is a frequent paradigm. It is conceivable that CRSs Type 3 could precipitate ACS, stroke, or other acute cardiac event; however, the epidemiological evidence and pathophysiological basis are yet to be described. In summary, CRSs Type 3 is not well defined for individual CVD events such as ACS, stroke, cardiac rehospitalization, arrhythmias, pump failure, and cardiac death and thus is a future research topic in terms of describing the epidemiology and pathogenesis of this syndrome [8].

Preventive Approaches. The major management principle concerning this syndrome is intra- and extravascular volume control with either use of diuretics and forms of extracorporeal volume and solute removal (CRRT, ultrafiltration, hemodialysis). In the setting of AKI, prevention of left ventricular volume overload is critical to maintain adequate cardiac output and systemic perfusion and avoid the viscous downward spiral in both cardiac and renal function.

\subsection{Chronic Cardiorenal Syndrome (Type 4): CKD Leading} to the Progression of CVD and Death. There is a graded and independent association between the severity of CKD, assessed by baseline eGFR, and incident as well as prevalent CVD [2]. A meta-analysis of 39 studies (1,371,990 participants) found a significant, independent relationship between the severity of CKD and the risk for all-cause death [48]. In this review, cardiovascular deaths constituted over $50 \%$ of fatalities. Thirteen studies have reported on the occurrence of CRSs Type 4 mainly in populations with ESRD on dialysis [8]. In this scenario, decreased renal function influences CVD outcomes in CRSs Type 4 by making conventional management of CAD or HF more difficult $[49,50]$. Azotemia and hyperkalemia are known to limit the use of drugs that antagonize the renin-angiotensin aldosterone system; thus, fewer patients with CKD enjoy the cardiovascular benefits of angiotensin converting enzyme inhibitors, angiotensin II receptor antagonists, and aldosterone receptor blockers [51, 52]. The presence of CKD also increases the severity, worsens the response to treatment, and is associated with poor cardiac and renal outcomes in acute and chronic hypertension [53, 54]. Amongst clinicians, the perceived risks of AKI in patients with CKD generally produce more conservative management strategies which have been associated with poor outcomes in the setting of ACS and stable CVD [55].

Preventive Approaches. Optimal treatment of CKD with blood pressure and glycemic control, RAAS blockers, and disease-specific therapies, when indicated, are the best means of preventing this syndrome. Morbidities of CKD, including bone and mineral disorder and anemia, should be managed according to CKD guidelines; however, clinical trials have failed to demonstrate that treatment of these problems influences CVD outcomes [56, 57].

2.5. Secondary Cardiorenal Syndrome (Type 5): Systemic Illness Leading to Simultaneous Heart and Renal Failure. It is recognized that a systemic insult, particularly in a younger patient with no prior heart or kidney disease, can lead to simultaneous organ dysfunction. This is almost always in the setting of critical illness such as sepsis, multiple trauma, or burns and can be thought of being part of multiorgan system failure. There are limited data on the incidence and determinants of CRSs (Type 5) in part because confounders such as hypotension, respiratory failure, liver failure, and other organ injury beyond the cardiac and renal systems create a difficult human model for investigation. Sepsis as a precipitator of CRSs Type 5 is common, and its incidence is increasing, with a mortality estimated between $20 \%$ and $60 \%$ [58-60]. Approximately $11-64 \%$ of septic patients develop AKI that is associated with a higher morbidity and mortality [61]. Abnormalities in cardiac function are also common in sepsis including wall motion abnormalities and transient reductions in left ventricular ejection fraction [62]. Observational data have found approximately $30-80 \%$ of individuals with sepsis have measurable blood troponin I or $\mathrm{T}$ that elevates above the 99th detection limits [63]. These elevated cardiac biomarkers have been associated with reduced left ventricular function and higher mortality even in patients without known coronary disease [64, 65]. Importantly, volume overload as a result of aggressive fluid resuscitation appears to be a significant determinant of CRSs Type 5. Among the 3,147 patients enrolled in the Sepsis Occurrence in Acutely Ill Patients (SOAP), there was a 36\% incidence of AKI, and volume overload was the strongest predictor of mortality [66]. Iatrogenic volume overload appears to play an important additional role, possibly along the lines described for CRSs Type 1 and passive venous congestion of the kidney, in the pathogenesis of AKI. At the same time, volume overload increases left ventricular wall tension and likely contributes to cardiac decompensation in those predisposed to both systolic and diastolic HF [67]. In summary for CRSs Type 5, both AKI and markers of cardiac injury followed by volume overload are common in sepsis, with each being associated with increased mortality. However, there is a current lack of integrative information on the incidence of bidirectional organ failure and its pathophysiological correlates in a variety of acute care settings.

Preventive Approaches. There are no proven methods to prevent or ameliorate this form of CRSs at this time. Randomized trials of early versus later intervention with CRRT have shown no differential benefit. Supportive care with a judicious intravenous fluid approach and the use of pressor agents as needed to avoid hypotension are reasonable but cannot be expected to avoid AKI or cardiac damage [68]. 


\section{Biomarkers of Cardiorenal Syndromes}

There is considerable interest in blood and urine biomarkers to detect CRSs. For decades, the rise in serum creatinine has been the only detectable sign of a reduction in glomerular filtration. Creatinine has had the disadvantages of being linked to creatine and the overall body muscle mass, hence, differing according to body size in addition to the rate of renal elimination [69]. Furthermore, the kidney both filters and secretes creatinine. Finally, the assays used to measure creatinine have not been standardized across laboratories; therefore, studies reporting values from multiple centers have inherent variation in values attributed to differences in measurement technique [70]. Hence, there is a clear need for better laboratory markers of renal filtration. An ideal marker would be independent of muscle mass, reflect actual renal filtration as the time it was measured, and be sensitive to changes in actual glomerular filtration rate (GFR) in order to signal clinicians to a meaningful change shortly after it occurs.

Unlike cardiac biomarkers indicating myocardial injury and overload (troponin, creatine kinase myocardial band, and natriuretic peptides), the field of nephrology has been devoid of approved blood or urine markers of AKI. Thus, the current paradigm is that when renal injury occurs, clinicians must wait to observe a reduction in GFR before AKI is inferred. The concept of measuring makers of the acute injury process is crucial to the early upstream identification of AKI before there is serious loss of organ function [71]. Below is a summary of relatively novel renal markers and what is known about them in acute cardiac and renal injury. Their use in the years to come will undoubtedly influence the epidemiology of CRSs. However, there are pitfalls to the widespread use of novel biomarkers including inappropriate conclusions along all lines of clinical decision making. Thus, considerable data are needed before any new marker enters the clinical arena.

3.1. Catalytic Iron. Iron is the most common metal element in the human body, and there are elaborate transport and management systems for its use in a variety of critical cellular systems including oxygen transport and cellular respiration. It has been known that poorly liganded iron is the critical basis for the generation of the hydroxyl radical, which is the most destructive of all reactive oxygen species. Using the bleomycin detectable assay, Lele and coworkers have recently demonstrated the release of catalytic iron into the blood in patients with acute coronary syndromes [72]. In this study, the appearance of catalytic iron preceded the rise in serum troponin and had an area under the receiver operating characteristic curve for the detection of acute myocardial infarction over 0.90. Labile iron is also believed to play an essential role in the oxidative organ damage of AKI as discussed above [73]. It is believed that local cellular and tissue availability of catalytic iron determines the degree and severity of organ injury in the setting of most hypoxic and other toxic insults [74]. Thus, catalytic iron may serve as both a diagnostic and therapeutic target by using iron chelators in the future for CRSs [75].
3.2. Neutrophil Gelatinase-Associated Lipocalin (NGAL). Siderocalin, or NGAL, was originally identified as a $25 \mathrm{kDa}$ protein which is a natural siderophore which works to scavenge cellular and pericellular labile iron, and thus, reducing its availability for bacterial growth. By reducing the availability of poorly liganded $\mathrm{Fe}(\mathrm{II})$ and $\mathrm{Fe}(\mathrm{III})$, which are needed to catalyze the Haber-Weiss and Fenton equations in the generation of reactive oxygen species, NGAL appears to have an important role in limiting oxidative damage in both acute and chronic diseases. NGAL seems to be one of the earliest kidney markers of ischemic or nephrotoxic injury in animal models, and it may be detected in the blood and urine of humans soon after AKI. Several studies have confirmed these findings; in intensive care adult patients with AKI secondary to sepsis, ischemia, or nephrotoxins, NGAL is significantly increased in the plasma and urine when compared to normal controls [76].

3.3. Cystatin C. Cystatin $\mathrm{C}$ is a cysteine protease inhibitor that is synthesized and released into the blood at a relatively constant rate by all nucleated cells. It is freely filtered by the glomerulus, completely reabsorbed by the proximal tubule, and not secreted into urine. Its blood levels are not affected by age, gender, race, or muscle mass; thus, it appears to be a better predictor of glomerular function than serum creatinine in patients with CKD. In AKI, urinary excretion of cystatin $\mathrm{C}$ has been shown to predict the requirement for renal replacement therapy earlier than creatinine. Finally, cystatin $\mathrm{C}$ has consistently outperformed serum creatinine and eGFR in the risk prediction for events in patient with CVD [77].

3.4. Kidney Injury Molecule 1 (KIM-1). Kidney Injury Molecule 1 (KIM-1) is a transmembrane glycoprotein which is not normally detectable in urine [78]. KIM-1 is measurable in the urine after ischemic or nephrotoxic insults to proximal tubular cells [79]. Urinary KIM-1 seems to be highly specific for AKI due to systemic illnesses such as sepsis and not for prerenal azotemia or drug-induced renal injury. Importantly, KIM-1 may be elevated before there is histologic evidence of proximal tubular cell death [44].

3.5. N-Acetyl- $\beta$ - $(D)$ Glucosaminidase (NAG). Recognized over thirty years ago, NAG is a lysosomal brush border enzyme found in proximal tubular cells. It is a large protein $(>130 \mathrm{kD})$ and is therefore not filtered through the glomerular membrane. NAG has been shown to function as a marker of AKI, reflecting particularly the degree of tubular damage. It is not only found in elevated urinary concentrations in AKI and CKD but also in diabetic patients, patients with essential hypertension and heart failure [80].

3.6. Interleukin-18 (IL-18). IL-18 is a proinflammatory cytokine detected in the urine after acute ischemic proximal tubular damage [81]. It displays sensitivity and specificity for ischemic AKI with an area under the receiver operating characteristic curve of $>90 \%$ with increased levels 48 hours prior to increase of serum creatinine. It has been associated 
with AKI mortality, but like other interleukins, it is not organ specific. IL-18 has also been theorized to participate in myocardial cell damage in the setting of ACS, and inhibitors of IL-18 expressed by stem cells have been shown to be protective in models of myocyte injury [82].

3.7. Liver Fatty Acid-Binding Protein (L-FABP). Liver fatty acid-binding protein (L-FABP) binds selectively to intracellular free unsaturated fatty acids and lipid peroxidation products during hypoxic tissue injury and is found in the urine of patients with AKI [83]. Urinary L-FABP is a potential biomarker for the detection and assessment of AKI and may be useful in predicting dialysis-free survival [84].

3.8. Tubular Enzymuria. Isoforms of a variety of enzymes released from proximal and renal tubular cells are measurable in the urine. These include gamma glutamyl transpeptidase (GGT), alkaline phosphatase, lactate dehydrogenase, and $\alpha$ and $\pi$ glutathione S-transferase (GST) [85-87]. It is possible that a panel of these markers measure in the urine could give important internal validity to not only the presence of, but potentially the location of injury along the nephron [88].

\section{Conclusions}

This paper has summarized a newly proposed framework for CRSs in order to better understand five possible subtypes [3]. A description of possible heart-kidney interactions is critical to our understanding and will guide future investigations into pathophysiology, screening, diagnosis, prognosis, and management. Recent studies have identified and characterized several novel biomarkers for CRSs. It is anticipated that these biomarkers will help make an earlier diagnosis of CRSs as well as identify its specific type and potentially its pathophysiology. Of particular interest is the recognition that tiny amounts of poorly bound labile iron catalyze oxidative stress reactions and further propagate organ injury. This may be a final common mechanism for most CRSs variants, and thus a prime diagnostic and therapeutic target in future clinical study. It is hoped in the future that some of these new biomarkers including catalytic iron and the kidney's response, NGAL, will provide sufficient risk prediction and early diagnosis to allow for prevention and treatment CRSs. It remains to be seen whether or not effective prevention and treatment of CRSs will improve hard renal and cardiac outcomes including ESRD, hospitalizations, and death.

\section{References}

[1] P. A. McCullough, "Why is chronic kidney disease the "spoiler" for cardiovascular outcomes?" Journal of the American College of Cardiology, vol. 41, no. 5, pp. 725-728, 2003.

[2] M. J. Sarnak, A. S. Levey, A. C. Schoolwerth et al., "Kidney disease as a risk factor for development of cardiovascular disease: a statement from the American Heart Association Councils on Kidney in Cardiovascular Disease, High Blood Pressure Research, Clinical Cardiology, and Epidemiology and Prevention," Circulation, vol. 108, no. 17, pp. 2154-2169, 2003.
[3] C. Ronco, P. McCullough, S. D. Anker et al., "Cardio-renal syndromes: report from the consensus conference of the acute dialysis quality initiative," European Heart Journal, vol. 31, no. 6, pp. 703-711, 2010.

[4] S. V. Shah, "Oxidants and iron in chronic kidney disease," Kidney International, Supplement, vol. 66, no. 91, pp. S50-S55, 2004.

[5] B. L. Walker, J. W. C. Tiong, and W. A. Jefferies, "Iron metabolism in mammalian cells," International Review of Cytology, vol. 211, pp. 241-278, 2001.

[6] D. R. Richardson, D. J.R. Lane, E. M. Becker et al., "Mitochondrial iron trafficking and the integration of iron metabolism between the mitochondrion and cytosol," Proceedings of the National Academy of Sciences of the United States of America, vol. 107, no. 24, pp. 10775-10782, 2010.

[7] J. Balla, G. M. Vercellotti, V. Jeney et al., "Heme, heme oxygenase, and ferritin: how the vascular endothelium survives (and dies) in an iron-rich environment," Antioxidants and Redox Signaling, vol. 9, no. 12, pp. 2119-2137, 2007.

[8] S. M. Bagshaw, D. N. Cruz, N. Aspromonte et al., "Epidemiology of cardio-renal syndromes: workgroup statements from the 7th ADQI Consensus Conference," Nephrology Dialysis Transplantation, vol. 25, no. 5, pp. 1406-1416, 2010.

[9] A. Levin, J. A. Kellum, and R. L. Mehta, "Acute Kidney Injury Network (AKIN). Acute kidney injury: toward an integrated understanding through development of a research agenda," Clinical Journal of the American Society of Nephrology, vol. 3, no. 3, pp. 862-863, 2008.

[10] D. E. Forman, J. Butler, Y. Wang et al., "Incidence, predictors at admission, and impact of worseningrenal function among patients hospitalized with heart failure," Journal of the American College of Cardiology, vol. 43, no. 1, pp. 61-67, 2004.

[11] M. R. Cowie, M. Komajda, T. Murray-Thomas, J. Underwood, and B. Ticho, "Prevalence and impact of worsening renal function in patients hospitalized with decompensated heart failure: results of the prospective outcomes study in heart failure (POSH)," European Heart Journal, vol. 27, no. 10, pp. 1216-1222, 2006.

[12] M. Metra, S. Nodari, G. Parrinello et al., "Worsening renal function in patients hospitalised for acute heart failure: clinical implications and prognostic significance," European Journal of Heart Failure, vol. 10, no. 2, pp. 188-195, 2008.

[13] J. M. Testani, J. Chen, B. D. McCauley, S. E. Kimmel, and R. P. Shannon, "Potential effects of aggressive decongestion during the treatment of decompensated heart failure on renal function and survival," Circulation, vol. 122, no. 3, pp. 265$272,2010$.

[14] W. H. Tang and W.. Mullens, "Cardiorenal syndrome in decompensated heart failure," Heart, vol. 96, no. 4, pp. 255260, 2010.

[15] P. A. McCullough, "Contrast-induced acute Kidney injury," Journal of the American College of Cardiology, vol. 51, no. 15, pp. 1419-1428, 2008.

[16] R. Bellomo, S. Auriemma, A. Fabbri et al., "The pathophysiology of cardiac surgery-associated acute kidney injury (CSAAKI)," International Journal of Artificial Organs, vol. 31, no. 2, pp. 166-178, 2008.

[17] M. M. Elahi, N. R. Battula, N. S. Hakim, and B. M. Matata, "Acute renal failure in patients with ischemic heart disease: causes and novel approaches in breaking the cycle of self-perpetuating insults abrogated by surgery," International Surgery, vol. 90, no. 4, pp. 202-208, 2006.

[18] M. Haase, R. Bellomo, and A. Haase-Fielitz, "Novel biomarkers, oxidative stress, and the role of labile iron toxicity 
in cardiopulmonary bypass-associated acute kidney injury," Journal of the American College of Cardiology, vol. 55, no. 19, pp. 2024-2033, 2010.

[19] C. Diez, A. Haneya, F. Brünger et al., "Minimized extracorporeal circulation cannot prevent acute kidney injury but attenuates early renal dysfunction after coronary bypass grafting," ASAIO Journal, vol. 55, no. 6, pp. 602-607, 2009.

[20] M. Ranucci, A. Ballotta, A. Kunkl et al., "Influence of the timing of cardiac catheterization and the amount of contrast media on acute renal failure after cardiac surgery," American Journal of Cardiology, vol. 101, no. 8, pp. 1112-1118, 2008.

[21] P. A. McCullough, R. Wolyn, L. L. Rocher, R. N. Levin, and W. W. O'Neill, "Acute renal failure after coronary intervention: incidence, risk factors, and relationship to mortality," American Journal of Medicine, vol. 103, no. 5, pp. 368-375, 1997.

[22] D. del Duca, S. Iqbal, E. Rahme, P. Goldberg, and B. de Varennes, "Renal failure after cardiac surgery : timing of cardiac catheterization and other perioperative risk factors," Annals of Thoracic Surgery, vol. 84, no. 4, pp. 1264-1271, 2007.

[23] B. B. Newsome, D. G. Warnock, W. M. McClellan et al., "Longterm risk of mortality and end-stage renal disease among the elderly after small increases in serum creatinine level during hospitalization for acute myocardial infarction," Archives of Internal Medicine, vol. 168, no. 6, pp. 609-616, 2008.

[24] P. A. McCullough, F. Stacul, C. R. Becker et al., "Contrastinduced nephropathy (CIN) Consensus Working Panel: executive summary," Reviews in Cardiovascular Medicine, vol. 7, no. 4, pp. 177-197, 2006.

[25] G. L. Smith, J. H. Lichtman, M. B. Bracken et al., "Renal impairment and outcomes in heart failure: systematic review and meta-analysis," Journal of the American College of Cardiology, vol. 47, no. 10, pp. 1987-1996, 2006.

[26] E. F. Philbin, P. A. McCullough, G. W. Dec, and T. G. DiSalvo, "Length of stay and procedure utilization are the major determinants of hospital charges for heart failure," Clinical Cardiology, vol. 24, no. 1, pp. 56-62, 2001.

[27] P. A. McCullough, "Acute kidney injury with iodinated contrast," Critical Care Medicine, vol. 36, no. 4, supplement, pp. S204-S211, 2008.

[28] R. M. Mentzer Jr., M. C. Oz, R. N. Sladen et al., "Effects of perioperative nesiritide in patients with left ventricular dysfunction undergoing cardiac surgery:the NAPA Trial," Journal of the American College of Cardiology, vol. 49, no. 6, pp. 716-726, 2007.

[29] C. Ronco, D. Cruz, and R. Bellomo, "Continuous renal replacement in critical illness," Contributions to Nephrology, vol. 156, pp. 309-319, 2007.

[30] K. Kundhal and C. E. Lok, "Clinical epidemiology of cardiovascular disease in chronic kidney disease," Nephron Clinical Practice, vol. 101, no. 2, pp. c47-c52, 2005.

[31] K. M. Chinnaiyan, D. Alexander, and P. A. McCullough, "Role of angiotensin II in the evolution of diastolic heart failure," Journal of Clinical Hypertension), vol. 7, no. 12, pp. 740-747, 2005.

[32] M. W. Yerkey, S. J. Kernis, B. A. Franklin, K. R. Sandberg, and P. A. McCullough, "Renal dysfunction and acceleration of coronary disease," Heart, vol. 90, no. 8, pp. 961-966, 2004.

[33] P. A. McCullough, S. Li, C. T. Jurkovitz et al., "Chronic kidney disease, prevalence of premature cardiovascular disease, and relationship to short-term mortality," American Heart Journal, vol. 156, no. 2, pp. 277-283, 2008.

[34] P. A. McCullough, K. R. Sandberg, F. Dumler, and J. E. Yanez, "Determinants of coronary vascular calcification in patients with chronic kidney disease and end-stage renal disease: a systematic review," Journal of Nephrology, vol. 17, no. 2, pp. 205-215, 2004.

[35] Y. Tintut and L. L. Demer, "Recent advances in multifactorial regulation of vascular calcification," Current Opinion in Lipidology, vol. 12, no. 5, pp. 555-560, 2001.

[36] P. A. McCullough, V. Agrawal, E. Danielewicz, and G. S. Abela, "Accelerated atherosclerotic calcification and mönckeberg's sclerosis: a continuum of advanced vascular pathology in chronic kidney disease," Clinical Journal of the American Society of Nephrology, vol. 3, no. 6, pp. 1585-1598, 2008.

[37] A. R. Guérin, B. Pannier, F. Métivier, S. J. Marchais, and G. M. London, "Assessment and significance of arterial stiffness in patients with chronic kidney disease," Current Opinion in Nephrology and Hypertension, vol. 17, no. 6, pp. 635-641, 2008.

[38] H. E. Bays, "“Sick fat," metabolic disease, and atherosclerosis," American Journal of Medicine, vol. 122, no. 1, pp. S26-S37, 2009.

[39] A. Ahmed, M. W. Rich, P. W. Sanders et al., "Chronic kidney disease associated mortality in diastolic versus systolic heart failure: a propensity matched study," American Journal of Cardiology, vol. 99, no. 3, pp. 393-398, 2007.

[40] S. S. Soman, K. R. Sandberg, S. Borzak, M. P. Hudson, J. Yee, and P. A. McCullough, "The independent association of renal dysfunction and arrhythmias in critically ill patients," Chest, vol. 122, no. 2, pp. 669-677, 2002.

[41] K. Jenkins and M. Kirk, "Heart failure and chronic kidney disease: an integrated care approach," Journal of Renal Care, vol. 36, supplement 1, pp. 127-135, 2010.

[42] A. Wase, A. Basit, R. Nazir et al., "Impact of chronic kidney disease upon survival among implantable cardioverterdefibrillator recipients," Journal of Interventional Cardiac Electrophysiology, vol. 11, no. 3, pp. 199-204, 2004.

[43] R. J. Glassock, R. Pecoits-Filho, and S. H. Barberato, "Left ventricular mass in chronic kidney disease and ESRD," Clinical Journal of the American Society of Nephrology, supplement 1, pp. S79-S91, 2009.

[44] M. Kobayashi, N. Hirawa, S. Morita et al., "Silent brain infarction and rapid decline of kidney function in patients with CKD: a prospective cohort study," American Journal of Kidney Diseases, vol. 56, no. 3, pp. 468-476, 2010.

[45] V. Agrawal, A. Shah, C. Rice, B. A. Franklin, and P. A. McCullough, "Impact of treating the metabolic syndrome on chronic kidney disease," Nature Reviews Nephrology, vol. 5, no. 9, pp. 520-528, 2009.

[46] V. Agrawal, V. Marinescu, M. Agarwal, and P. A. McCullough, "Cardiovascular implications of proteinuria: an indicator of chronic kidney disease," Nature reviews. Cardiology, vol. 6, no. 4, pp. 301-311, 2009.

[47] S. M. Bagshaw and D. N. Cruz, "Fluid overload as a biomarker of heart failure and acute kidney injury," Contributions to Nephrology, vol. 164, pp. 54-68, 2010.

[48] M. Tonelli, N. Wiebe, B. Culleton et al., "Chronic kidney disease and mortality risk: a systematic review," Journal of the American Society of Nephrology, vol. 17, no. 7, pp. 2034-2047, 2006.

[49] M. G. Shlipak, "Pharmacotherapy for heart failure in patients with renal insufficiency," Annals of Internal Medicine, vol. 138, no. 11, pp. 917-924, 2003.

[50] P. A. McCullough and A. D. Berman, "Percutaneous coronary interventions in the high-risk renal patient: strategies for renal protection and vascular protection," Cardiology Clinics, vol. 23, no. 3, pp. 299-310, 2005.

[51] P. A. McCullough, K. R. Sandberg, J. Yee, and M. P. Hudson, "Mortality benefit of angiotensin-converting enzyme 
inhibitors after cardiac events in patients with end-stage renal disease," JRAAS - Journal of the Renin-Angiotensin-Aldosterone System, vol. 3, no. 3, pp. 188-191, 2002.

[52] P. A. McCullough, "Chronic kidney disease: tipping the scale to the benefit of angiotensin-converting enzyme inhibitors in patients with coronary artery disease," Circulation, vol. 114, no. 1, pp. 6-7, 2006.

[53] R. Kalaitzidis, S. Li, C. Wang, S.-C. Chen, P. A. McCullough, and G. L. Bakris, "Hypertension in early-stage kidney disease: an update from the Kidney Early Evaluation Program (KEEP)," American Journal of Kidney Diseases, vol. 53, supplement 4, pp. S22-S31, 2009.

[54] L. A. Szczech, C. B. Granger, J. F. Dasta et al., "Acute kidney injury and cardiovascular outcomes in acute severe hypertension," Circulation, vol. 121, no. 20, pp. 2183-2191, 2010.

[55] E. C. Keeley, R. Kadakia, S. Soman, S. Borzak, and P. A. McCullough, "Analysis of long-term survival after revascularization in patients with chronic kidney disease presenting with acute coronary syndromes," American Journal of Cardiology, vol. 92, no. 5, pp. 509-514, 2003.

[56] S. C. Palmer, S. D. Navaneethan, J. C. Craig et al., "Metaanalysis: erythropoiesis-stimulating agents in patients with chronic kidney disease," Annals of Internal Medicine, vol. 153, no. 1, pp. 23-33, 2010.

[57] P. A. McCullough and K. M. Chinnaiyan, "Annual progression of coronary calcification in trials of preventive therapies a systematic review," Archives of Internal Medicine, vol. 169, no. 22, pp. 2064-2070, 2009.

[58] D. C. Angus, W. T. Linde-Zwirble, J. Lidicker, G. Clermont, J. Carcillo, and M. R. Pinsky, "Epidemiology of severe sepsis in the United States: analysis of incidence, outcome, and associated costs of care," Critical Care Medicine, vol. 29, no. 7, pp. 1303-1310, 2001.

[59] G. S. Martin, D. M. Mannino, S. Eaton, and M. Moss, "The epidemiology of sepsis in the United States from 1979 through 2000," New England Journal of Medicine, vol. 348, no. 16, pp. 1546-1554, 2003.

[60] S. M. Bagshaw, S. Lapinsky, S. Dial et al., "Acute kidney injury in septic shock: clinical outcomes and impact of duration of hypotension prior to initiation of antimicrobial therapy," Intensive Care Medicine, pp. 1-11, 2008.

[61] J. A. Lopes, S. Jorge, C. Resina et al., "Acute renal failure in patients with sepsis," Critical Care, vol. 11, no. 2, article 411, 2007.

[62] S. L. Zanotti-Cavazzonia and S. M. Hollenberg, "Cardiac dysfunction in severe sepsis and septic shock," Current Opinion in Critical Care, vol. 15, no. 5, pp. 392-397, 2009.

[63] R. Favory and R. Neviere, "Significance and interpretation of elevated troponin in septic patients," Critical Care, vol. 10, no. 4, article no. 224, 2006.

[64] P. Ammann, M. Maggiorini, O. Bertel et al., "Troponin as a risk factor for mortality in critically ill patients without acute coronary syndromes," Journal of the American College of Cardiology, vol. 41, no. 11, pp. 2004-2009, 2003.

[65] N. J. Mehta, I. A. Khan, V. Gupta, K. Jani, R. M. Gowda, and P. R. Smith, "Cardiac troponin I predicts myocardial dysfunction and adverse outcome in septic shock," International Journal of Cardiology, vol. 95, no. 1, pp. 13-17, 2004.

[66] J.-L. Vincent, Y. Sakr, C. L. Sprung et al., "Sepsis in European intensive care units: results of the SOAP study," Critical Care Medicine, vol. 34, no. 2, pp. 344-353, 2006.

[67] K. M. Chinnaiyan, D. Alexander, M. Maddens, and P. A. McCullough, "Curriculum in cardiology: integrated diagnosis and management of diastolic heart failure," American Heart Journal, vol. 153, no. 2, pp. 189-200, 2007.

[68] J. R. Prowle and R. Bellomo, "Continuous renal replacement therapy: recent advances and future research," Nature Reviews Nephrology, vol. 6, no. 9, pp. 521-529, 2010.

[69] G. L. Myers, W. G. Miller, J. Coresh et al., "Recommendations for improving serum creatinine measurement: a report from the Laboratory Working Group of the National Kidney Disease Education Program," Clinical Chemistry, vol. 52, no. 1, pp. 5$18,2006$.

[70] L. A. Stevens and N. Stoycheff, "Standardization of serum creatinine and estimated GFR in the Kidney Early Evaluation Program (KEEP)," American Journal of Kidney Diseases, vol. 51, no. 4, supplement, pp. S77-S82, 2008.

[71] S. S. Soni, C. Ronco, N. Katz, and D. N. Cruz, "Early diagnosis of acute kidney injury: the promise of novel biomarkers," Blood Purification, vol. 28, no. 3, pp. 165-174, 2009.

[72] S. Lele, S. Shah, P. A. McCullough, and M. Rajapurkar, "Serum catalytic iron as a novel biomarker of vascular injury in acute coronary syndromes," EuroIntervention, vol. 5, no. 3, pp. 336342, 2009.

[73] R. Baliga, N. Ueda, P. D. Walker, and S. V. Shah, "Oxidant mechanisms in toxic acute renal failure," Drug Metabolism Reviews, vol. 31, no. 4, pp. 971-997, 1999.

[74] D. B. Kell, "Iron behaving badly: inappropriate iron chelation as a major contributor to the aetiology of vascular and other progressive inflammatory and degenerative diseases," $B M C$ Medical Genomics, vol. 2, article 2, 2009.

[75] M. R. Moussavian, J. E. Slotta, O. Kollmar, M. D. Menger, G. Gronow, and M. K. Schilling, "Post-hypoxic cellular disintegration in glycine-preserved renal tubules is attenuated by hydroxyl radical scavengers and iron chelators," Langenbeck's Archives of Surgery, vol. 393, no. 3, pp. 303-310, 2008.

[76] K. Mori and K. Nakao, "Neutrophil gelatinase-associated lipocalin as the real-time indicator of active kidney damage," Kidney International, vol. 71, no. 10, pp. 967-970, 2007.

[77] M. D. McMurray, J. E. Trivax, and P. A. McCullough, "Serum cystatin C, renal filtration function, and left ventricular remodeling," Circulation. Heart failure, vol. 2, no. 2, pp. 8689, 2009.

[78] W. C. Prozialeck, J. R. Edwards, P. C. Lamar, J. Liu, V. S. Vaidya, and J. V. Bonventre, "Expression of kidney injury molecule-1 (Kim-1) in relation to necrosis and apoptosis during the early stages of Cd-induced proximal tubule injury," Toxicology and Applied Pharmacology, vol. 238, no. 3, pp. 306-314, 2009.

[79] V. S. Vaidya, V. Ramirez, T. Ichimura, N. A. Bobadilla, and J. V. Bonventre, "Urinary kidney injury molecule-1: a sensitive quantitative biomarker for early detection of kidney tubular injury," American Journal of Physiology — Renal Physiology, vol. 290, no. 2, pp. F517-F529, 2006.

[80] J. M. Wellwood, B. G. Ellis, R. G. Price, K. Hammond, A. E. Thompson, and N. F. Jones, "Urinary N-acetyl- betaD-glucosaminidase activities in patients with renal disease," British Medical Journal, vol. 3, no. 5980, pp. 408-411, 1975.

[81] C. R. Parikh, E. Abraham, M. Ancukiewicz, and C. L. Edelstein, "Urine IL-18 is an early diagnostic marker for acute kidney injury and predicts mortality in the intensive care unit," Journal of the American Society of Nephrology, vol. 16, no. 10, pp. 3046-3052, 2005.

[82] M. Wang, J. Tan, Y. Wang, K. K. Meldrum, C. A. Dinarello, and D. R. Meldrum, "IL-18 binding protein-expressing mesenchymal stem cells improve myocardial protection after ischemia or infarction," Proceedings of the National Academy of Sciences 
of the United States of America, vol. 106, no. 41, pp. 1749917504, 2009.

[83] E. Noiri, K. Doi, K. Negishi et al., "Urinary fatty acid-binding protein 1: an early predictive biomarker of kidney injury," American Journal of Physiology—Renal Physiology, vol. 296, no. 4, pp. F669-F679, 2009.

[84] B. A. McMahon and P. T. Murray, "Urinary liver fatty acidbinding protein: another novel biomarker of acute kidney injury," Kidney International, vol. 77, no. 8, pp. 657-659, 2010.

[85] A. A. Banday, N. Farooq, S. Priyamvada, A. N. K. Yusufi, and F. Khan, "Time dependent effects of gentamicin on the enzymes of carbohydrate metabolism, brush border membrane and oxidative stress in rat kidney tissues," Life Sciences, vol. 82, no. 9-10, pp. 450-459, 2008.

[86] B. Lisowska-Myjak, "Serum and urinary biomarkers of acute kidney injury," Blood Purification, vol. 29, no. 4, pp. 357-365, 2010.

[87] Z. H. Endre and J. Westhuyzen, "Early detection of acute kidney injury: emerging new biomarkers," Nephrology, vol. 13, no. 2, pp. 91-98, 2008.

[88] X.-L. Liang, S.-X. Liu, Y.-H. Chen et al., "Combination of urinary kidney injury molecule-1 and interleukin-18 as early biomarker for the diagnosis and progressive assessment of acute kidney injury following cardiopulmonary bypass surgery: a prospective nested casecontrol study," Biomarkers, vol. 15, no. 4, pp. 332-339, 2010. 


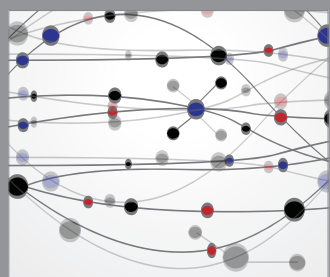

The Scientific World Journal
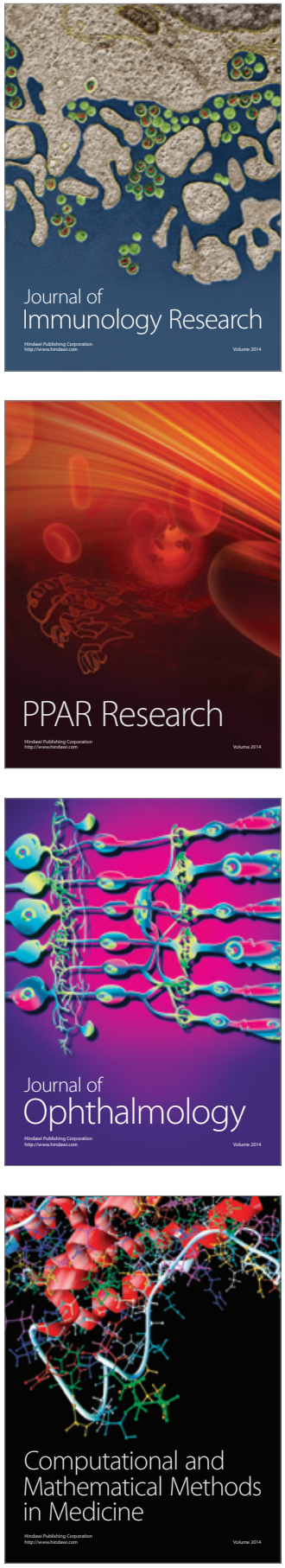

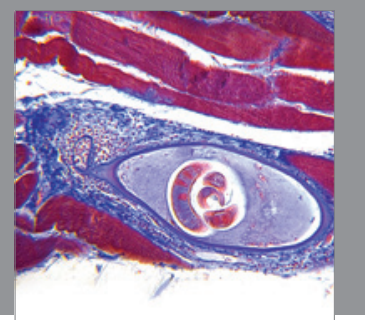

Gastroenterology

Research and Practice
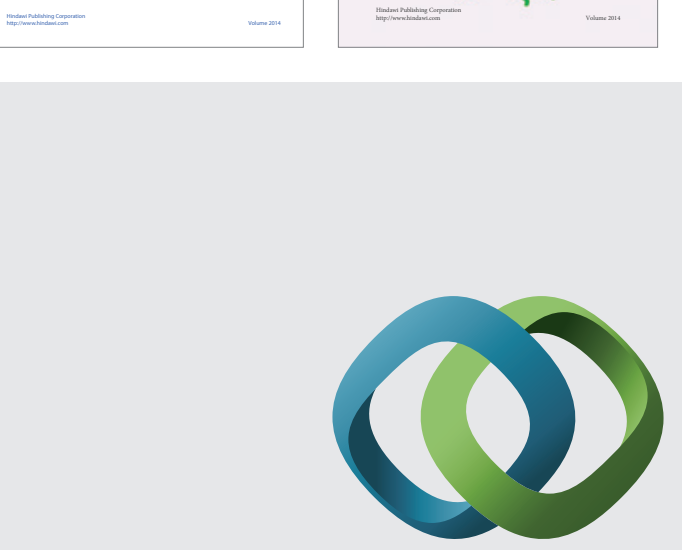

\section{Hindawi}

Submit your manuscripts at

http://www.hindawi.com
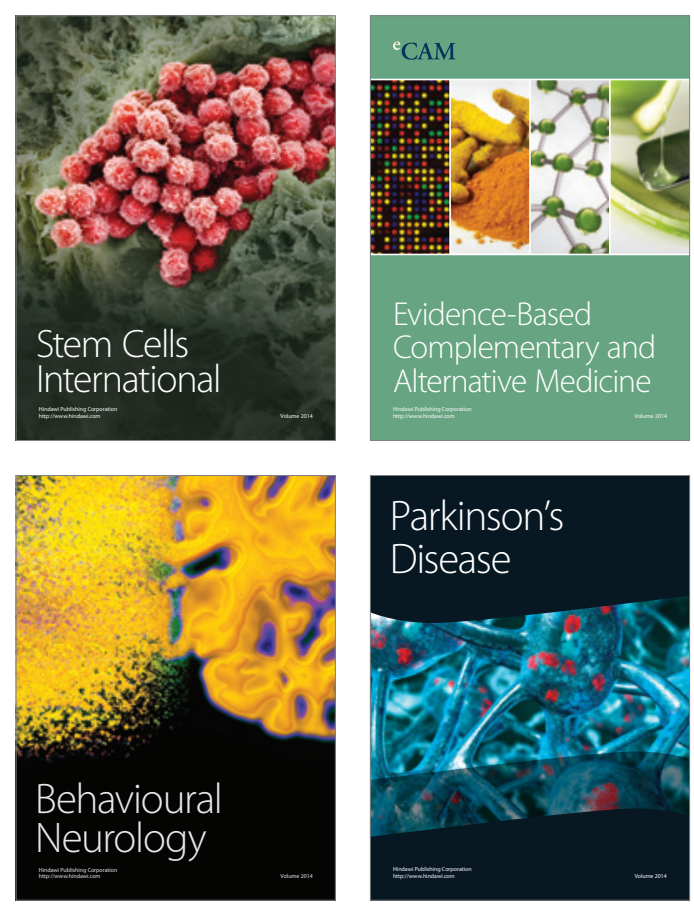

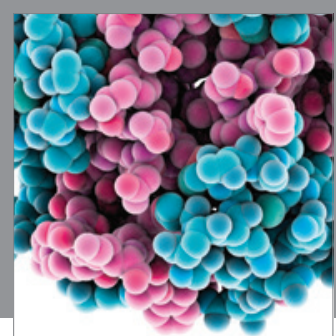

Journal of
Diabetes Research

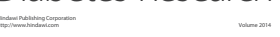

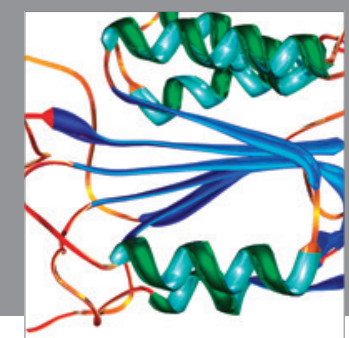

Disease Markers
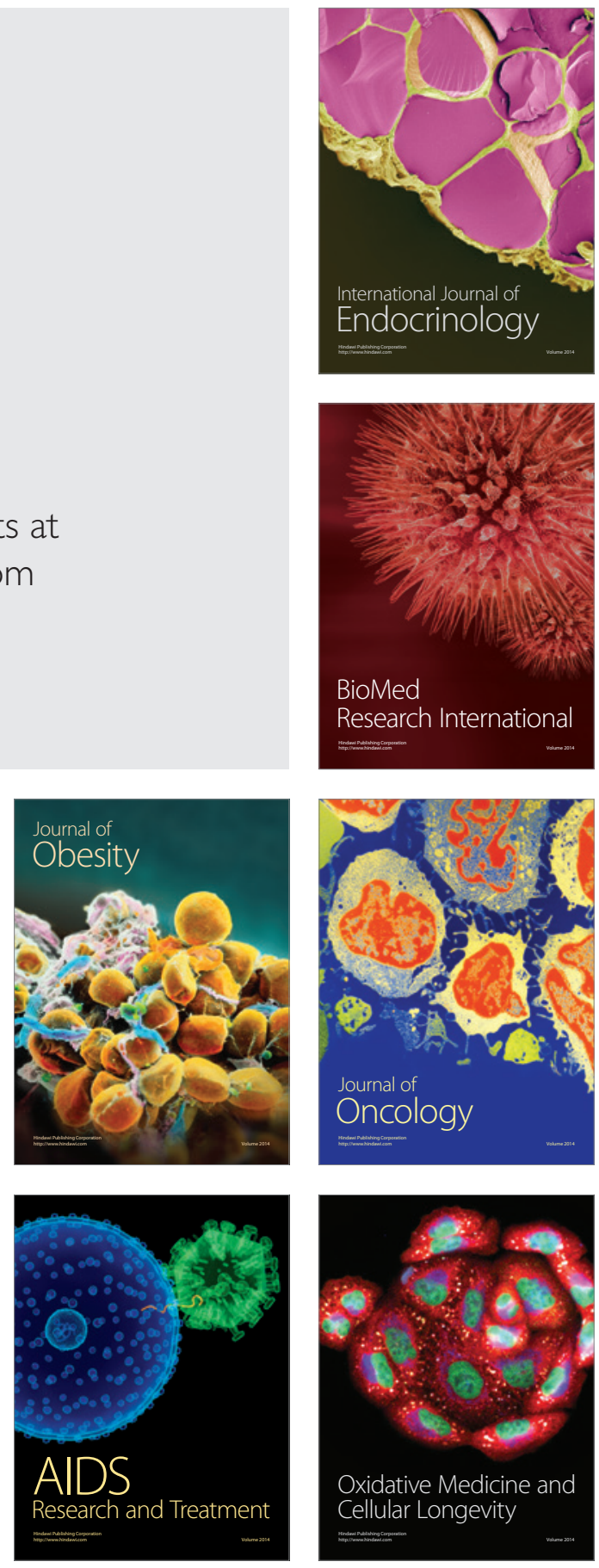